\title{
Postantibiotic effect of roxithromycin, erythromycin, and clindamycin against selected Gram-positive bacteria and Haemophilus influenzae
}

\author{
B. Kaenzi, Ch. Segessenmann and A. U. Gerber \\ Regionalspital Burgdorf and University of Bern, Switzerland
}

\begin{abstract}
Recent experimental work has shown that a so-called PAE (postantibiotic effect, i.e. persistent suppression of regrowth after short exposure of bacteria to the study drug in vitro) is a feature of most current antibiotics. However, marked quantitative differences were found between different types of antibiotics and also between Gram-positive and Gram-negative organisms studied. A PAE has not yet been demonstrated for roxithromycin, a new macrolide antibiotic. Therefore, we compared the PAE of roxithromycin, erythromycin, and clindamycin against laboratory strains and clinical isolates of Staphylococcus aureus, Streptococcus pyogenes, Str. pnewmoniae, and Haemophilus influenzae in vitro. Identical multiples of the MIC and identical exposure times resulted in similar PAEs for the three study drugs tested. Good correlations could be found between the area under the in-vitro concentration-vs-time curve (AUC) and PAEs. The longest PAE of $9.6 \mathrm{~h}$ was observed after exposure of Str. pneumoniae to $1.9 \mathrm{mg} / \mathrm{l}$ of roxithromycin for $6 \mathrm{~h}$.
\end{abstract}

\section{Introduction}

In recent experimental work Bundtzen et al. (1981) demonstrated that after short exposure of bacteria to antimicrobial agents a so-called postantibiotic effect (PAE) on bacterial regrowth, after short exposure to the study drug in vitro, is a feature of most current antibiotics. A good review of the current knowledge of PAE was recently published by Craig \& Gudmundsson (1986). Antibiotics acting by inhibiting protein synthesis exhibited a PAE at a low concentration whereas higher drug concentrations (in multiples of the MIC) were needed to obtain a significant PAE with $\beta$-lactam drugs.

In the present study, we compared the PAE of roxithromycin, erythromycin, and clindamycin against selected respiratory pathogens. Study organisms were laboratory strains and recent clinical isolates of Staphylococcus aureus, Streptococcus pyogenes. Str. pneumoniae, and Haemophilus influenzae. Some of the clinical strains were isolated from patients with lower respiratory tract infections before they were treated successfully with roxithromycin.

\section{Materials and methods}

Organisms. Staph. aureus ATCC 29213, and Str. pneumoniae ATCC 27336 were the standard study organisms. Additional strains of Staph. aureus, Str. pneumoniae,

Correspondence: Andreas U. Gerber, M.D., Medizinische Abteilung, Regionalspital, 3400 Burgdorf, Switzerland.

Supported by the Swiss National Foundation for Scientific Research (Grant No 3.907-083) and a limited grant from Roussel-Uclaf, Paris, France. 
Str. pyogenes, and $H$. influenzae from clinical sources were included in confirmatory studies.

Media. Different media were used for specific organisms: trypticase soy broth (TSB, Difco Laboratories, Detroit, Michigan) for staphylococci; Schaedler broth (BBL, Microbiology Systems, Cockeysville, Madison) for streptococci; brain-heart infusion broth (Difco) supplemented with $5 \%$ Fildes' enrichement (BBL) for $H$. influenzae. All media were supplemented with $50 \mathrm{mg} / \mathrm{l}$ of calcium and $25 \mathrm{mg} / 1$ of magnesium as recommended by Stratton \& Reller (1977).

Bacterial inocula. All studies were done with organisms in the logarithmic phase of growth at the time of exposure to antibiotic. To obtain such a culture a single colony was inoculated into the appropriate medium and incubated at $37^{\circ} \mathrm{C}$ for approximately $8 \mathrm{~h}$ before each experiment. When the OD at $580 \mathrm{~nm}$ reached 0.3 , the culture was diluted 1:10 (1:100 for $H$. influenzae) in control medium. This procedure resulted in a final concentration of approximately $10^{7} \mathrm{cfu} / \mathrm{ml}$. This point was defined as time 0 of exposure to antibiotic. Counts of cfu were performed immediately to check the original inoculum.

Antibiotics. Stock solutions of 1 and $3.2 \mathrm{~g} / \mathrm{l}$ were prepared from standard powders obtained from the manufacturers. Roxithromycin was supplied by Roussel-Uclaf, Paris, France, erythromycin by Abbott Laboratories, Queenborough, Kent, England, and clindamycin by Upjohn Co., Zurich, Switzerland.

MICs were repeatedly determined by the microtitre technique according to Jones et al. (1985). Antibiotic concentrates were diluted in the appropriate medium so that the desired concentration was achieved for each experiment.

Removal of antibiotics. After incubation of the cultures at $37^{\circ} \mathrm{C}$ for $1-6 \mathrm{~h}$, the antibiotic was removed by a $10^{-3}$ dilution into fresh medium as previously described by Bundtzen et al. (1981). All media for subcultures were prewarmed to $37^{\circ} \mathrm{C}$.

Controls. During each experiment, the following controls were maintained: (i) cultures prepared and treated as above, but without exposure to antibiotics and (ii) cultures prepared as above and continually exposed over 1,2 , and $6 \mathrm{~h}$ to antibiotics. By adequate dilution and supplementation control subcultures and pre-exposed subcultures maintained the same bacterial inoculum and calculated residual (carryover) drug concentration. Standard dilution of controls was $10^{-4}$.

Counts of cfu. Counts of colony forming units were made on trypticase soy agar (API bio Merieux, Geneva, Switzerland) for staphylococci; on trypticase soy agar with $5 \%$ sheep blood (API bio Merieux) for streptococci; and on chocolate agar with chemically defined enrichment (API bio Merieux) for $H$. influenzae. Sterile cold $0.9 \%$ $\mathrm{NaCl}$ was used for serial ten-fold dilution of cultures as required. A 10- $\mu$ l sample of each dilution was plated in duplicate on octants of the agar surface. Plates were read after incubation for $18-48 \mathrm{~h}$ at $37^{\circ} \mathrm{C}$ (incubation in an atmosphere of increased $\mathrm{CO}_{2}$ for streptococci and $H$. influenzae).

Counts of cfu were made for all cultures at time 0 , before and after removal of antibiotic, and every 1 or $2 \mathrm{~h}$ thereafter until either turbidity became marked or cultures remąined clear for $24 \mathrm{~h}$.

Quantitation of PAEs. The following calculations were used to quantitate the PAEs of the three study drugs and compare similar experiments performed on different days. (i) $T$ is the time required for the count of the cfu in the test culture to increase by 1 $\log _{10}$ above the count immediately after dilution. (ii) $C$ is the time required for the count of cfu in the drug-free control cultures to increase by $1 \log _{10}$ above the count 
immediately after dilution. (iii) $T$ minus $C$ expresses the time interval during which the tested drug is seen to affect bacterial growth after exposure. This calculation defined as the duration of PAE was used as a basis for comparison of persistent effects on the antibiotics studied.

In the main experiments identical inocula were exposed at the same time to two different concentrations of the three study drugs, using a range of $0.5-1 \times \mathrm{MIC}$ and 5-10 $\times$ MIC. For Str. pneumoniae an additional concentration of roxithromycin corresponding to 50-100 $\times$ MIC was included. Exposure times were 1, 2 and $6 \mathrm{~h}$.

\section{Results}

\section{MICs}

Table I gives a comparative summary of the MICs of 22 study organisms. Repeated determinations were carried out during the study. There was no significant difference of MICs in the test media compared to Mueller-Hinton broth. Staphylococci (number of tested strains $n=6)$, Str. pneumoniae $(n=8)$, and Str. pyogenes $(n=2)$ were all susceptible to the three study drugs, whereas $H$. influenzae $(n=4)$ showed marginal or no inhibition by roxithromycin, and cross resistance to erythromycin.

\section{Minimal concentrations affecting growth of low inocula}

Preliminary experiments showed that growth of small inocula $\left(10^{3}-10^{4} \mathrm{cfu} / \mathrm{ml}\right)$ of the test strains in $\log$ phase were not impaired by addition of up to $10^{-2} \mathrm{MIC}$ of the test drugs. Thus, a dilution of at least $10^{-3}$ was sufficient to eliminate drug action in the test culture, a method which had been confirmed in previous studies by Bundtzen et al. (1981).

\section{Variation of log-phase growth kinetics}

An analysis of all experiments performed with a given organism revealed a maximum variation of the initial 1 log-growth time of $23 \%$ among non-exposed control subcultures. Individual 1 log-growth times for small bacterial inocula of

Tabłe I. MICs of study organisms $(\mathrm{mg} / \mathrm{l})$

\begin{tabular}{lccc}
\hline Organism & Erythromycin & Clindamycin & Roxithromycin \\
\hline $\begin{array}{l}\text { Staph. aureus } \\
\text { ATCC 29213 } \\
\text { clinical isolates (6) }\end{array}$ & $\begin{array}{c}0.25-0.5 \\
0.5-1\end{array}$ & $0.06-0.25$ & $0.5-1$ \\
$\begin{array}{l}\text { Str. pyogenes } \\
\text { clinical isolates (2) }\end{array}$ & $0.06-0.25$ & $0.06-0.25$ & $0.5-2$ \\
$\begin{array}{l}\text { Str. pneumoniae } \\
\begin{array}{l}\text { ATCC 27336 } \\
\text { clinical isolates (8) }\end{array}\end{array}$ & $0.03-0.06$ & $0.03-0.06$ & $0.1-0.5$ \\
$\begin{array}{l}\text { H. infuenzae } \\
\text { clinical isolates (4) }\end{array}$ & $8-0.125$ & $0.06-0.125$ & $0.1-0.35$ \\
\hline
\end{tabular}

Number in parenthesis = number of organisms tested. 
$10^{3}-10^{4} \mathrm{cfu} / \mathrm{ml}$ were as follows: $1 \cdot 26 \mathrm{~h}$ (standard deviation (s.D.)) 0.05 ; calculated from nine curves) for Staph. aureus ATCC $29213 ; 2.35$ h (S.D. $0 \cdot 12 ; n=14$ ) for Str. pyogenes strain Abs 86-29; 1.43 h (S.D. 0.13; $n=9$ ) for Str. pneumoniae ATCC 27336, and $2.2 \mathrm{~h}$ (s.D. $0.14 ; n=15$ ) for $\beta$-lactamase-producing clinical isolates of $H$. influenzae.

\section{Time-kill curves}

In time-kill curves performed with inocula of $10^{7} \mathrm{cfu} / \mathrm{ml}$ all target organisms showed similar killing patterns for all three study drugs. Drug concentrations around the MIC showed mainly a bacteriostatic effect for the first $6-8 \mathrm{~h}$ followed by breakthrough growth. Concentrations from five to ten times the MIC exerted slow bactericidal killing of the chosen Gram-positive bacteria and $H$. influenzae, thus allowing most PAE determinations with appropriate $10^{-3}$ dilutions even after $6 \mathrm{~h}$ exposures.

\section{Stability of PAE}

Repetition of the same experiments on different days with slightly higher or lower inocula gave no more than an $8 \%$ variation in duration of the PAE. However, this finding was only valid for experiments performed with five to ten times the MIC. Marked variations (up to $50 \%$ ) were found after repeated experiments with drug concentrations near or under the MIC.

\section{$P A E$ of various species}

A typical PAE determination is shown in Figure 1. Str. pneumoniae ATCC 27336 was exposed for $2 \mathrm{~h}$ to $7 \mathrm{mg} / \mathrm{l}$ of roxithromycin (50-100 times the MIC). after which subcultures were started by diluting the drug-exposed and the control organisms into fresh drug-free broth. Analysis of regrowth curves showed a PAE of $5 \cdot 2 \mathrm{~h}$.

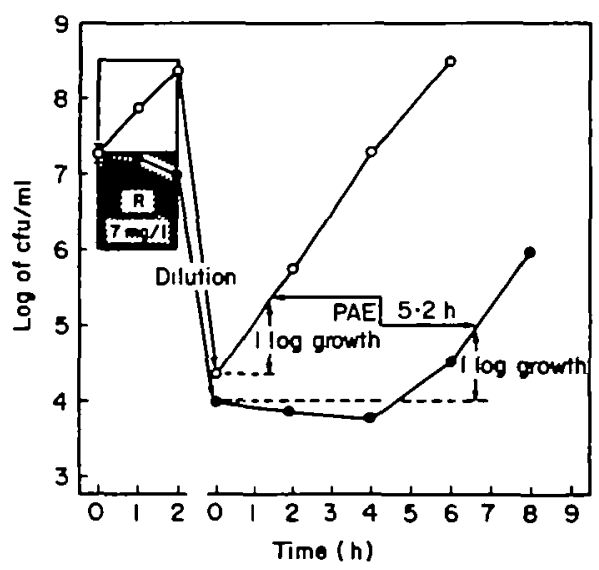

Figure 1. Postantobiotic effect (PAE) of roxithromycin (R) on Str. pneumoniae ATCC 27336. The duration of drug exposure was $2 \mathrm{~h}$; the drug concentration was $7 \mathrm{mg} / \mathrm{l}$ corresponding to $50-100$ times the MlC. The PAE is the difference in time to grow $1 \log _{10}$ (arrows) for the control culture $(O)$ and the pre-exposed culture $(O)$ as indicated. Initial $I \log$ growth time was 1.5 and $6.7 \mathrm{~h}$ for control and pre-exposed subcultures, respectively. 

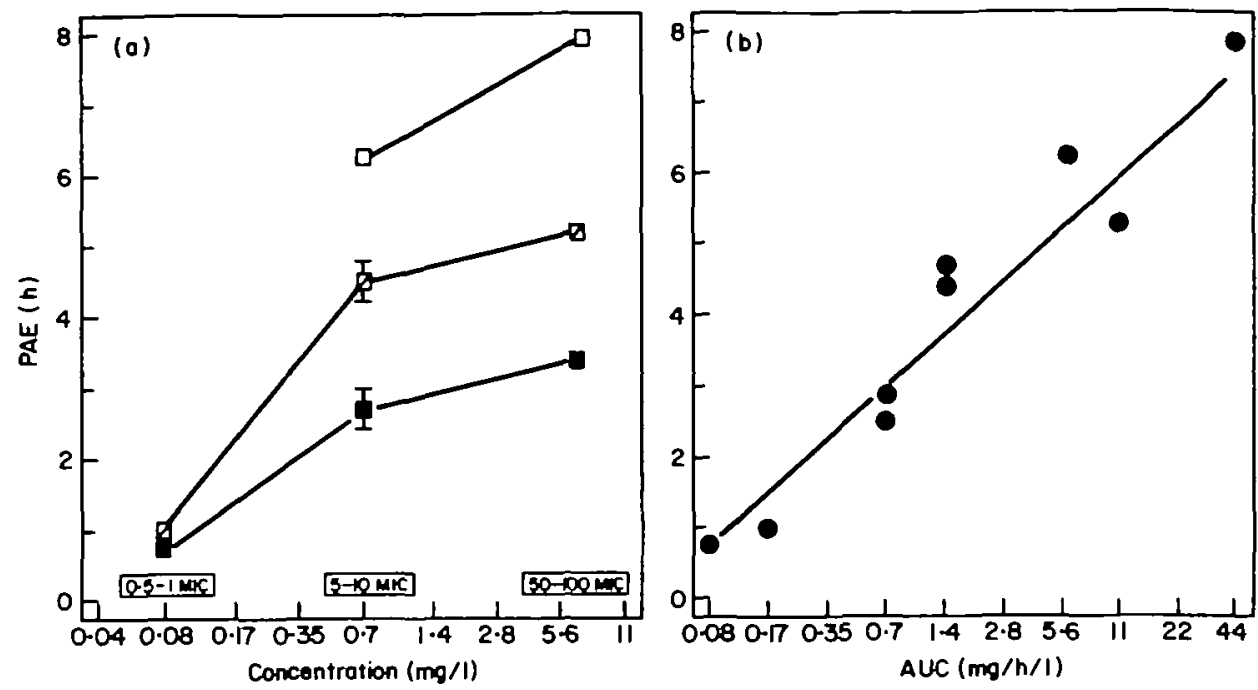

Figure 2. (a) PAE of roxithromycin on Str. pneumaniae as a function of drug concentration and exposure time (, $1 \mathrm{~h} ; \boldsymbol{\square}, 2 \mathrm{~h} ; \square, 6 \mathrm{~h})$. As for every investigated combination in this study the PAE correlated with both the drug concentration and the duration of drug exposure. (b) Correlation between the PAE and the in of the in-vitro AUC of roxithromycin on Str. preumoniae ATCC 27336. For nine PAE determinations the calculated correlation coefficient was $r=0.947$.

A series of additional experiments, showed that the PAE was dependent on both the concentration of the drug and the duration of drug exposure (Figure 2(a)). Two or three different drug concentrations and three different exposure times resulted in various areas under the in-vitro concentration vs. time curves (AUC). Therefore, the PAE was plotted against the ln of the in-vitro AUC of drug exposure. As shown in Figure 2(b) for roxithromycin, a good correlation was found $(r=0.947)$ up to a maximal response of $7.9 \mathrm{~h}$. Yet a shorter PAE than expected was found with combinations using high drug concentrations for short exposure times of 1-2 h (data not shown).

Similar experiments with erythromycin and clindamycin against Str. pneumoniae ATCC 27336 yielded similar results; i.e. identical multiples of the MIC and identical exposure times resulted in similar PAEs for the three study drugs tested. Results are shown in Figure 3. One hour exposures to the study drugs at concentration around the MIC resulted in PAEs for 1-2 h. In contrast, 1 and $2 \mathrm{~h}$ exposures to 5-10 $\times$ MIC were followed by PAEs as follows (in hours): erythromycin 3.2 and 5.3 ; clindamycin 2.5 and 4.9; roxithromycin 2.6 and 4.4 . Corresponding PAEs for a $6 \mathrm{~h}$ exposure were: erythromycin 6.3; clindamycin 6.9; roxithromycin 6.3. Concentrations of 50-100 times the MIC of roxithromycin produced a maximal PAE of $7.9 \mathrm{~h}$ after a $6 \mathrm{~h}$ exposure.

Against Str. pyogenes, a similar PAE of up to $1.5 \mathrm{~h}$ was observed after a $1 \mathrm{~h}$ and a $6 \mathrm{~h}$ exposure to $0.5-1 \mathrm{MIC}$ of the three study drugs. In contrast a $6 \mathrm{~h}$ exposure to 5-10 x MIC of erythromycin, clindamycin and roxithromycin resulted in PAEs of 6-7, 4.5-5.5 and 6-7 h, respectively. Results obtained with Str. pyogenes strain Abs 86-29 are shown in Figure 4. 


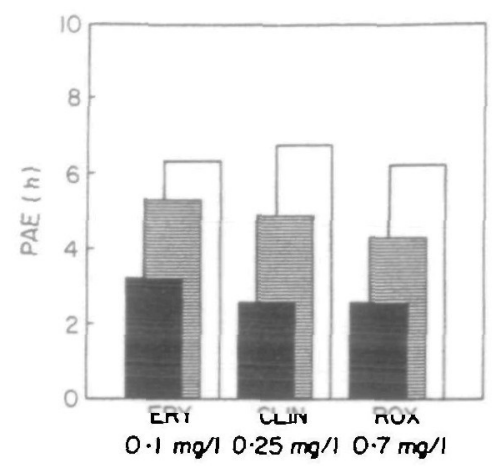

Figure 3. PAEs of 5-10 MICs of erythromycin (ERY), clindamycin (CLIN) and roxithromycin (ROX) on Sir. pneumoniae ATCC 27336. The used drug concentrations are indicated at the bottom of the colums. After three different exposure times $(\mathbb{D}, 1 \mathrm{~h} ; \mathrm{E}, 2 \mathrm{~h} ; \square, 6 \mathrm{~h})$ to similar multiples of the MIC each drug revealed a similar PAE, with a maximum PAE time of 6 to $7 \mathrm{~h}$ after a $6 \mathrm{~h}$ exposure.

Against Staph. aureus ATCC 29213 PAEs lasted 1.5 to $2.5 \mathrm{~h}$ after exposure to 0.5-1 MIC for 1-6h. Exposure to 5-10 $\times$ MIC produced PAEs from 2.5 to $5.2 \mathrm{~h}$ depending on exposure time. Individual results are shown in Figure 5. No major differences were found between the three study drugs on condition that PAEs of similar multiples of the MIC were studied.

PAEs obtained with recent clinical isolates of Str. pneumoniae and Staph. aureus were in the same range if identical MICs and exposure times of study drugs were compared. Absolute times of PAEs of the tested clinical isolates were even longer than those found with the standard strains. The longest PAE found with pneumococci was $9.57 \mathrm{~h}$ after a $6 \mathrm{~h}$ exposure to $1.9 \mathrm{mg} / \mathrm{l}$ of roxithromycin. PAEs of clinical isolates of staphylococci showed no major variations compared with the tested standard strain.

Against $H$. influenzae the PAE was insignificant after a one MIC exposure for $2 \mathrm{~h}$, yet a maximal PAE of 2-4 h was obtained after a 1-2 h exposure to ten MICs of erythromycin and roxithromycin. Ten MICs of clindamycin for 1-2 h produced a PAE of only $1-2 \mathrm{~h}$.

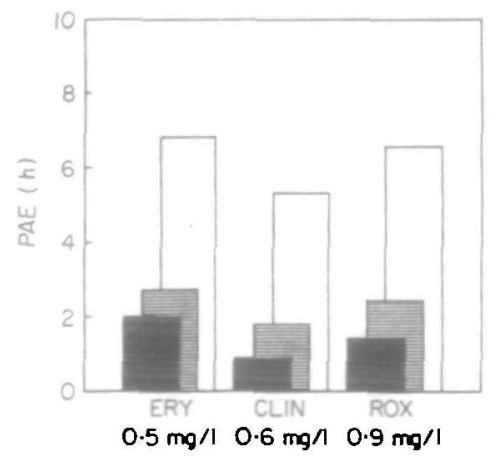

Figure 4. PAEs of 5-10 MICs of erythromycin (ERY), clindamycin (CLIN) and roxithromycin (ROX) on a clinical isolate of Str. pyogenes Abs 86-29. Results of exposure times of $1(\square), 2$ (E) and $6 \mathrm{~h}(\square)$ are shown. 


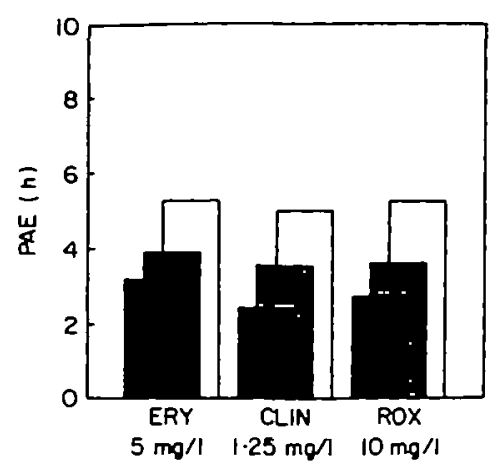

Figure 5. PAEs on Staph. aureus ATCC 29213 of 5-10 MICs of erythromycin (ERY), clindamycin (CLIN) and roxithromycin (ROX). Exposure times were $1(\square), 2$ (E) and $6 \mathrm{~h}(\square)$.

\section{Discussion}

Our present in-vitro study confirms and extends previous observations on the effect of short exposure of Gram-positive pathogens and $H$. influenzae to antimicrobial agents (Parker \& Marsh, 1946; Eagle \& Musselman, 1949; McDonald, Craig \& Kunin, 1977; Wilson \& Rolinson, 1979; Bundtzen et al., 1981; Gerber \& Craig, 1981). A PAE was demonstrated for three antimicrobial agents which act by inhibition of protein synthesis. Erythromycin has previously been shown to exert a PAE on respiratory pathogens (Gerber \& Craig 1981). A similar PAE was later demonstrated for clindamycin (Craig \& Gudmundsson, 1986). In the present paper we describe for the first time a PAE for the newest macrolide, roxithromycin.

For a given target organism the duration of the PAE was remarkably similar for all our three study drugs if comparable multiples of the MIC were used for a set duration of drug exposure. This finding was true not only for Gram-positive pathogens but also for $H$. influenzae. Thus, the capability of a given antibiotic to exert a PAE was linked to its intrinsic antimicrobial activity. This finding is consistent with previous PAE studies (Bundtzen et al., 1981; Gerber \& Craig 1981; Craig \& Gudmundsson, 1986). It can be explained, at least in part, by the fact that all three study drugs act by the same mechanism, namely binding to the S0S-subunit of bacterial ribosomes.

Despite its unique pharmacokinetic parameters in vivo and its long half life in particular, roxithromycin produced similar PAEs in vitro as erythromycin and clindamycin. Thus, the PAE as determined in vitro is not linked to its in-vivo pharmacokinetic parameters. On the other hand, it is important to note that in all experimental PAE work published so far the PAE depended on both the concentration of drug and the duration of drug exposure. As a consequence the PAE correlates also with the in-vitro $A U C$, i.e. concentration of drug in $\mathrm{mg} / \mathrm{l}$ multiplied by the duration of in-vitro exposure in hours (Bundtzen et al., 1981; Gerber \& Craig, 1982). In the present study the same correlation was shown for roxithromycin. Thus, trying to extrapolate these in-vitro facts to the in-vivo situation one would expect that the long in-vivo half life of roxithromycin would not only favour its antimicrobial activity but equally so its capability of exerting a PAE in vivo.

Against $H$. influenzae very high concentrations of our study drugs were needed to obtain a PAE. These concentrations were markedly higher than those which can be 
obtained by oral administration of these drugs in man. Thus, the PAE observed with these drugs in vitro is likely not to be obtained in vivo.

The PAE is a feature of almost any antimicrobial agent, and the presence or absence of a PAE has been used as an argument for dose spacing in antimicrobial chemotherapy. It is important to note, however, that still little is known about the PAE in vivo. Thus, a lot of additional work is needed both in vitro and in vivo including clinical studies to corroborate speculations on the significance of this phenomenon in respect to antimicrobial chemotherapy of infected patients.

\section{References}

Bundtzen, R. W., Gerber, A. U., Cohn, D. L. \& Craig, W. A. (1981). Postantibiotic suppression of baterial growth. Reviews of Infectious Diseases 3, 28-37.

Craig, W. A. \& Gudmundsson, S. (1986). The postantibiotic effect. In Antibiotics in Laboratory Medicine, 2nd edn (Lorian, V., Ed.), pp. 515-36. Williams \& Wilkins, Baltimore.

Eagle, H. \& Musselman, A. D. (1949). The slow recovery of bacteria from the toxic effects of penicillin. Journal of Bacteriology 58, 475-90.

Gerber, A. U. \& Craig, W. A. (1981). Growth kinetics of respiratory pathogens after short exposures to ampicillin and erythromycin in vitro. Journal of Antimicrobial Chemotherapy 8 , $81-91$.

Gerber, A. U. \& Craig, W. A. (1982). Experimentelle Studien zur Frage des optimalen Dosisintervalls in der Antibiotikatherapie. Schweizerische Medizinische Wochenschrift 112, 42-5.

Jones, R. N., Barry, A. L., Gavan, T. L. \& Washington, J. A. (1985). In Manual of Clinical Microbiology, 4th edn (Lenette, E. H., Balows, A., Hausler, W. J., Shadomy, H. J., Eds), pp. 972-77. American Society for Microbiology, Washington D.C.

Jones, R. N., Barry, A. L. \& Thornsberry, C. (1983). In vitro evaluation of three new macrolide antimicrobial agents, RU 28965, RU 29065, and RU 29702, and comparisons with other orally administered drugs. Antimicrobial Agents and Chemotherapy 24, 209-15.

McDonald, P. J., Craig, W. A. \& Kunin, C. M. (1977). Persistent effect of antibiotics on Staphylococcus aureus after exposure for limited periods of time. Journal of Infectious Diseases 135, 217-23.

Parker, R. F. \& Marsh, H. C. (1946). The action of penicillin on Staphylococcus. Journal of Bacteriology 51, 181-6.

Stratton, C. W. \& Reller, L. B. (1977). Serum dilution test for bactericidal activity. I. Selection of a physiologic diluent. Journal of Infectious Diseases 136, 187-95.

Wilson, D. A. \& Rolinson, G. N. (1979). The recovery period following exposure of bacteria to penicillin, Chemotherapy 25, 14-22. 